\title{
Range of motion in intervertebral joints: why we don't trust DinoMorph
}

\section{May 30, 2009}

Let's assume for a moment that you accept our contention (Taylor et al. 2009) that, since extant terrestrial tetrapods habitually hold their necks in maximal extension, sauropods did the same. That still leaves the question of why we have the neck of our Diplodocus reconstruction at a steep 45degree angle rather than the very gentle elevation that Stevens and Parrish's (1999) DinoMorph project permits.

As a reminder, here is fig. 6A of Stevens (2002), a paper on the computer science behind DinoMorph which used exactly the same models as the 1999 study but which conveniently illustrates them in lateral view:

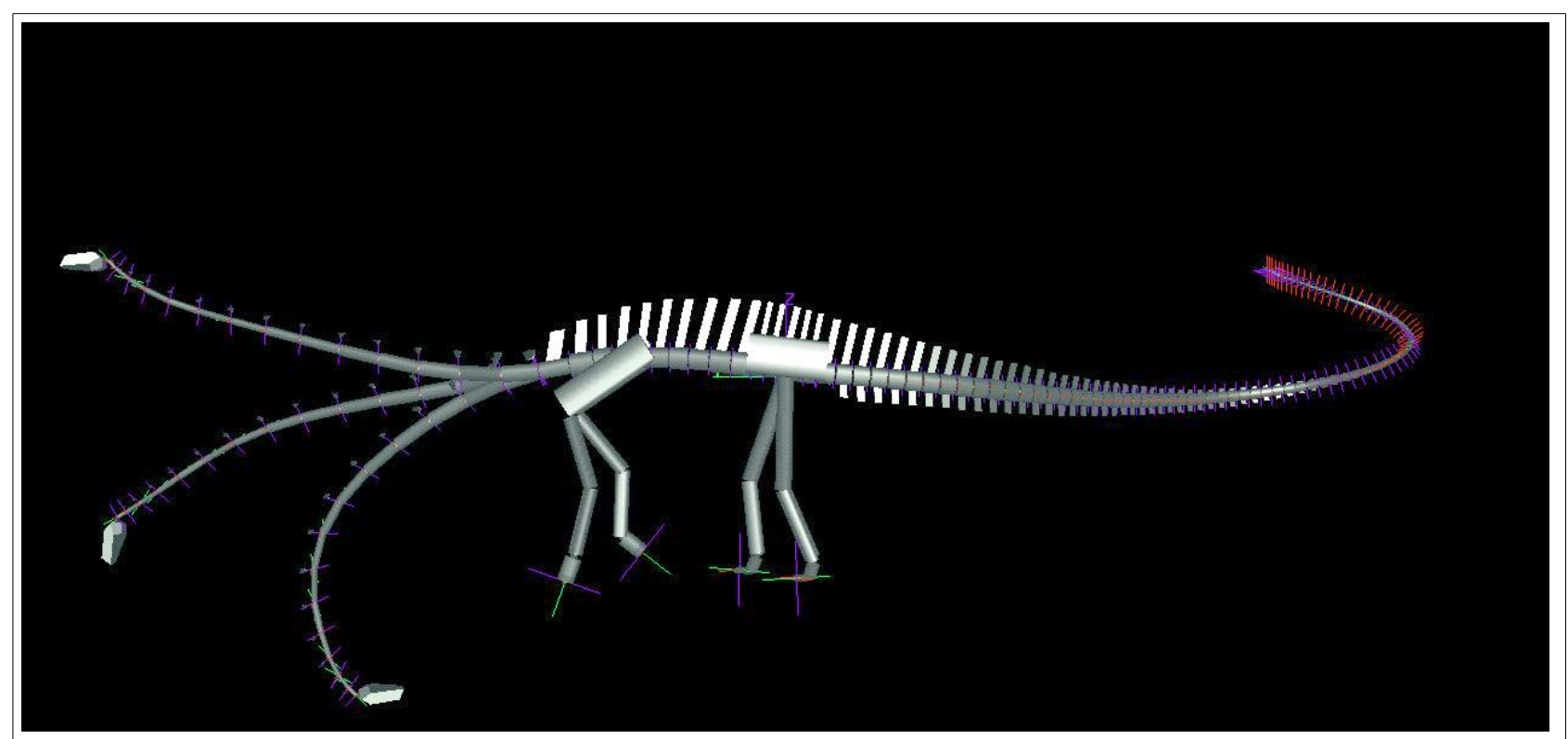

Stevens (2002: fig. 6A), illustrating the fully extended, neutral and fully flexed poses attainable by Diplodocus according to the original DinoMorph model. 
As you'll see, not only does the neutral pose show the characteristic subhorizontal neck with the drooping end, but even the maximally extended pose barely gets the head above the level of the back. In the most recent version of his Diplodocus model, Kent has slightly changed the angle at which the neck leaves the torso, due to a reconfiguration of the pectoral girdle, but this still leaves the neck very low.

So why did we do this?

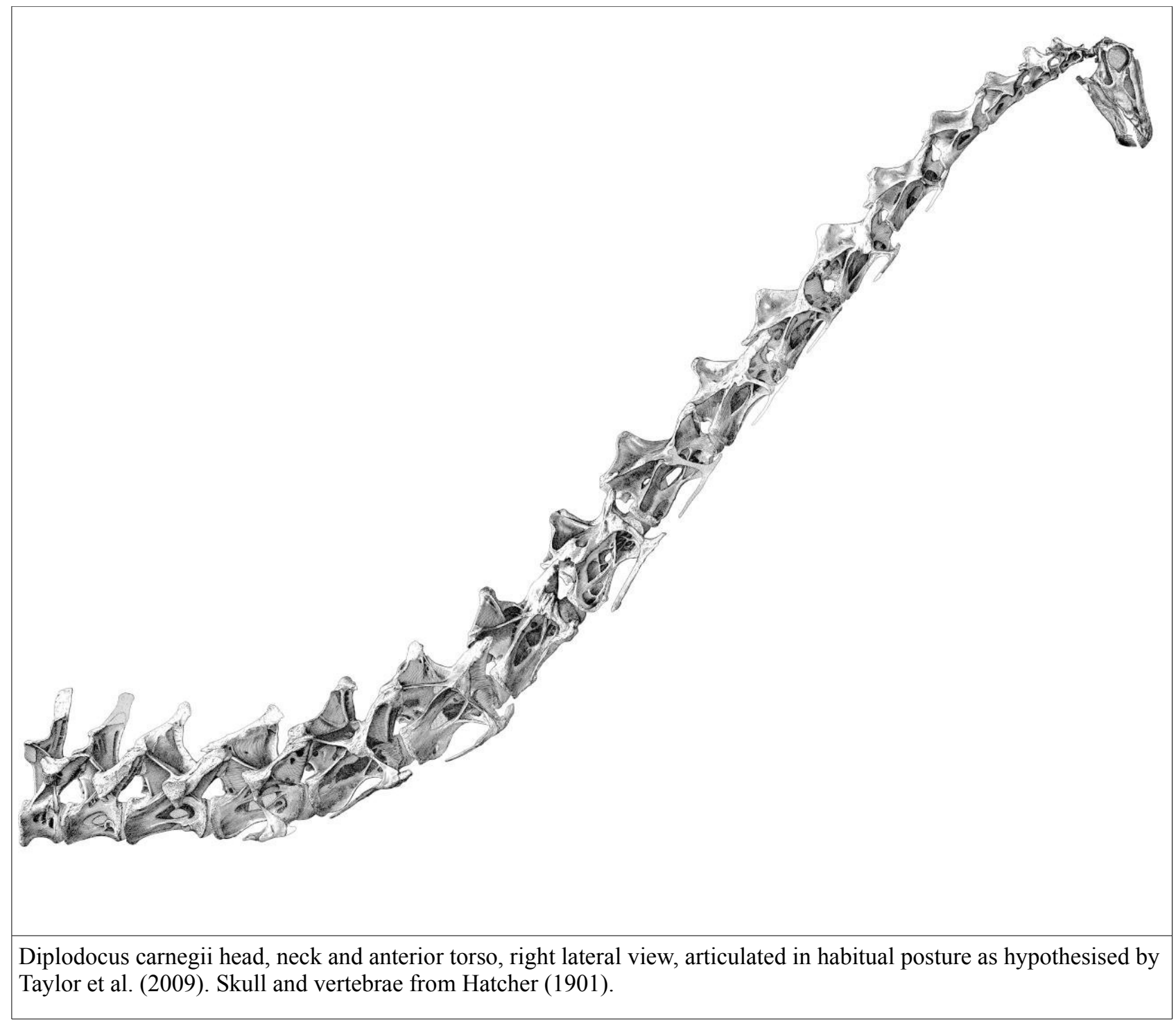

Doesn't the DinoMorph model show that the posterior cervicals just can't do this?

Well, maybe not. 
Remember that the precursor to the DinoMorph project was John Martin's (1987) paper on the mounting of the Rutland cetiosaur at the Leicester City Museum, in which he calculated neutral pose and the extreme extended and flexed poses by manipulating actual bones without the benefit of a computer. Martin ended up with a similar result to that Stevens and Parrish were later to get:

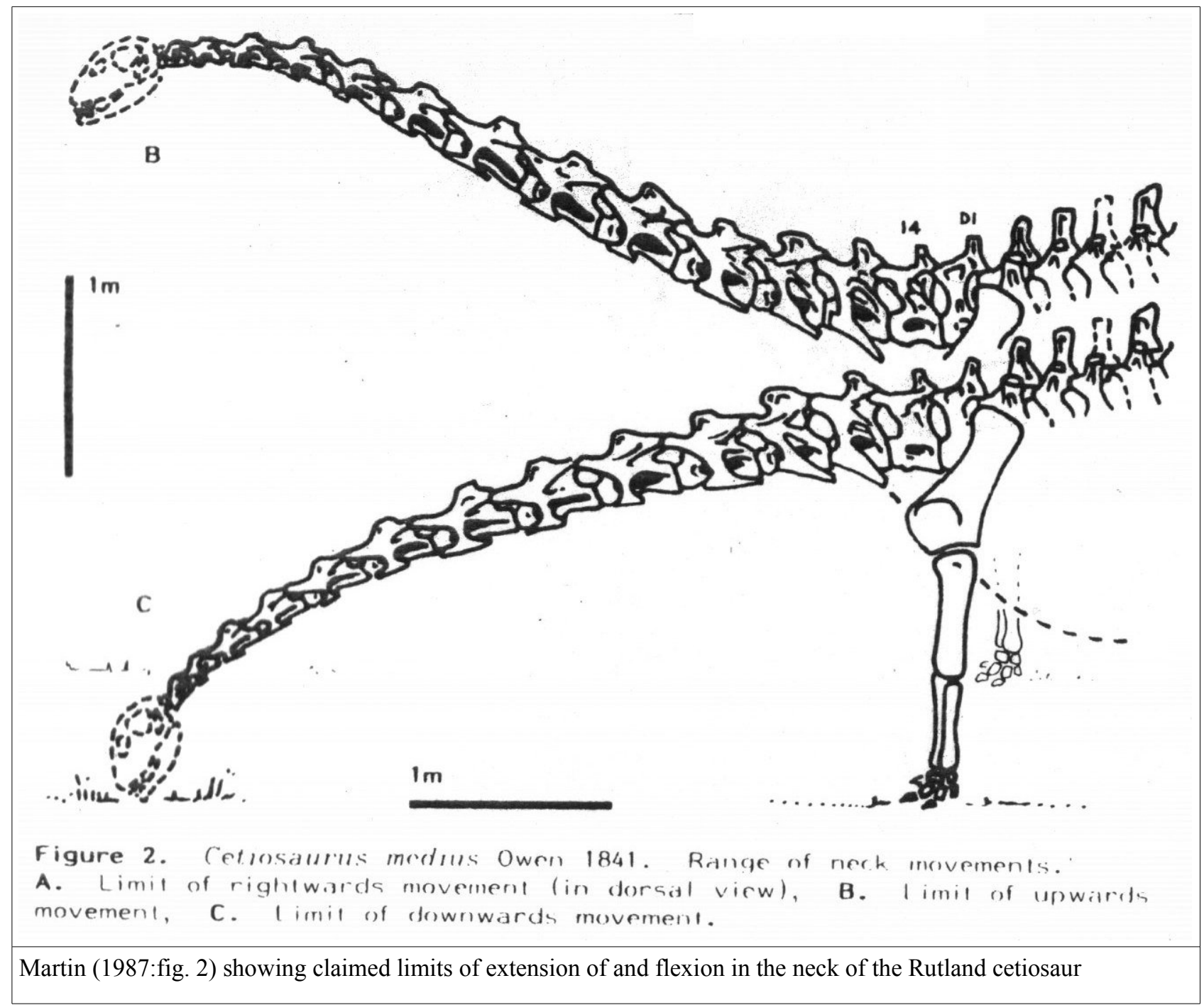


But when Matt and I looked at the actual mounted skeleton a few years back, what we saw didn't fit with this at all:

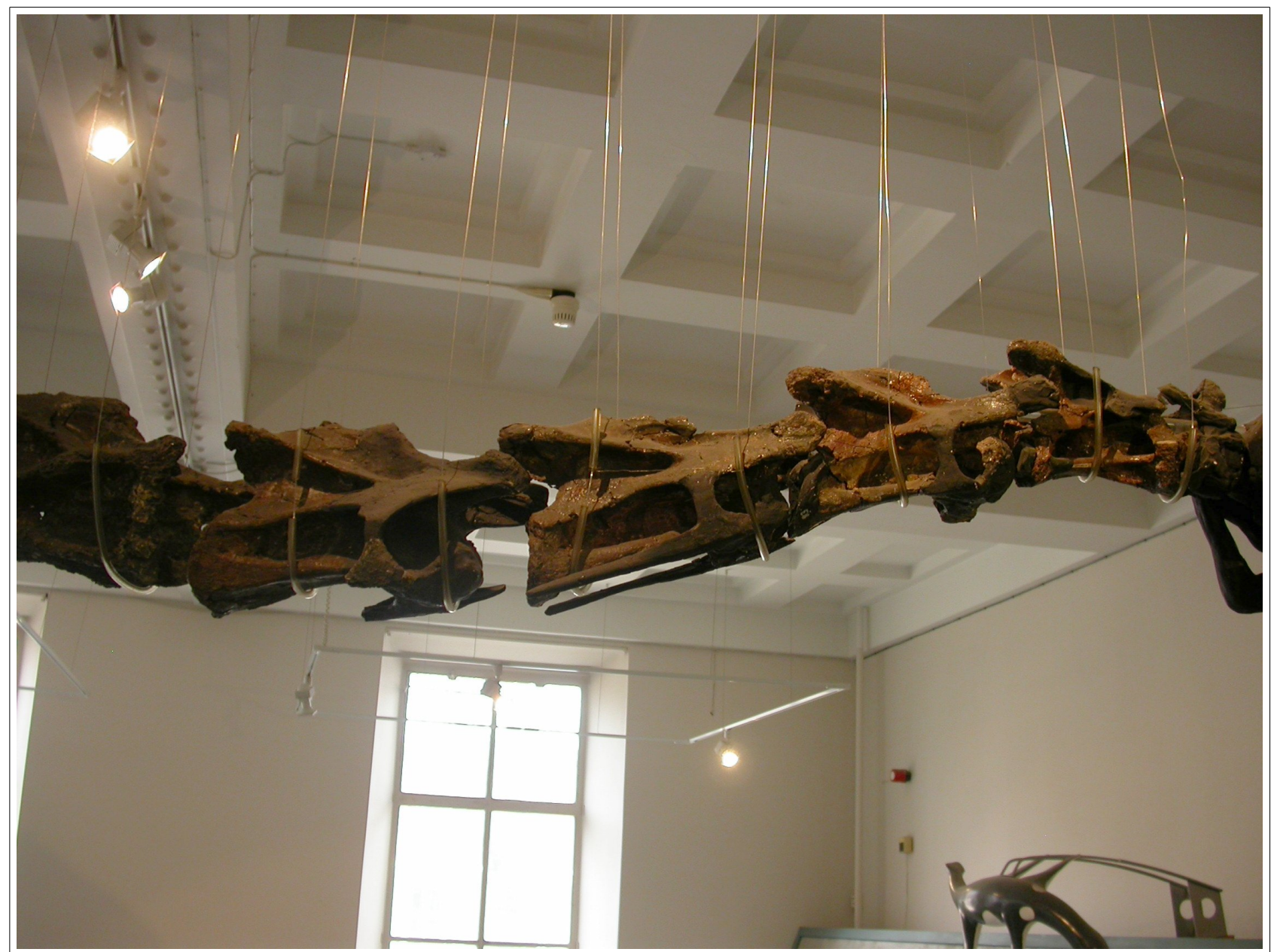

Rutland cetiosaur, anterior part of neck in right lateral view, showing extreme disarticulation between the cotyle of C4 and condyle of $\mathrm{C5}$

Check out that huge gap between the centra of the fourth and fifth cervicals! There's no way to avoid this without putting a comically extreme downward kink in the neck at this point. And there are similar gaps at other points along the neck, including some near the neck-base that would require a strong upward kink in order to articulate both the centra and the zygapophyses simultaneously.

Are we saying that in life, this specimen did have strong kinks in the neck? No, we're not (despite the pleasant coincidence that this would force the neck into an extreme version of the elevated pose we're advocating). What we're saying is that sauropod cervicals are rarely - I'd go so far as to say never - preserved undistorted, and so you just can't rely on how they seem to articulate, at least not for quantitative analyses. This post-mortem distortion should not be too surprising: unlike femora and other such solid bones, remember that the cervicals were highly pneumatic and composed primarily of laminae, which would be subject to all sorts of taphonomic and diagenetic distortion. In the extreme case of Sauroposeidon, the cervicals, which were up to $140 \mathrm{~cm}$ in length, "are of extremely light construction, with the outer layer of bone ranging in thickness from less than $1 \mathrm{~mm}$ (literally paper-thin) to approximately $3 \mathrm{~mm}$ " (Wedel et al. 2000:110-111) — it's astonishing that they are not much more smushed up than they are.

So Martin's cetiosaur seems too distorted to give meaningful articulation results, but what about the specimens that Stevens and Parrish used for the DinoMorph paper? Well, the Apatosaurus model is 
certainly based on questionable material. As pointed out by Upchurch (2000):

A second difficulty with Stevens and Parrish's analysis is that their data for Apatosaurus was derived from a single specimen in the Carnegie Museum (CM 3018). This generally well preserved specimen has suffered severe damage at the base of the neck, and the three most posterior cervicals are thus represented by plaster models that cannot provide reliable anatomical data (Gilmore 1936, pers. obs.). Although Stevens and Parrish acknowledge that the morphology of the posterior cervicals is particularly influential in determining the nature of the feeding envelope, they do not mention this problem, and it is not clear how this gap in the data was addressed in their analyses. This deficit could have had a profound impact on Stevens and Parrish's conclusions.

And Gilmore's observations are really rather damning: as well as the account of the damaged neckbase, he also noted (p. 195) that "the type of A. louisae [i.e. CM 3018] lacks most of the spine tops, only those of cervicals eight, ten and twelve being complete". (You would never guess this from Gilmore's Plate XXIV, which shows all of the cervicals but C5 essentially complete.) So all in all, the DinoMorph study's Apatosaurus is not one I'd want to build an argument on. 
What about the Diplodocus carnegii holotype CM 84, which is the Diplodocus used in the DinoMorph papers? That's just about the best preserved sauropod skeleton in the world, right? Well, yes. But even that is distorted enough that the neck can't be articulated without some sleight of hand. I don't have good photos of the mounted neck, unfortunately (and probably won't have until someone at the NHM gives me a stepladder and access to the holy of holies that surrounds the mount), but I did have the experience of photoshopping the cervical vertebra illustrations from Hatcher (1901: plate III) in an attempt to get them into a good pose, and I found that even these don't really fit properly:

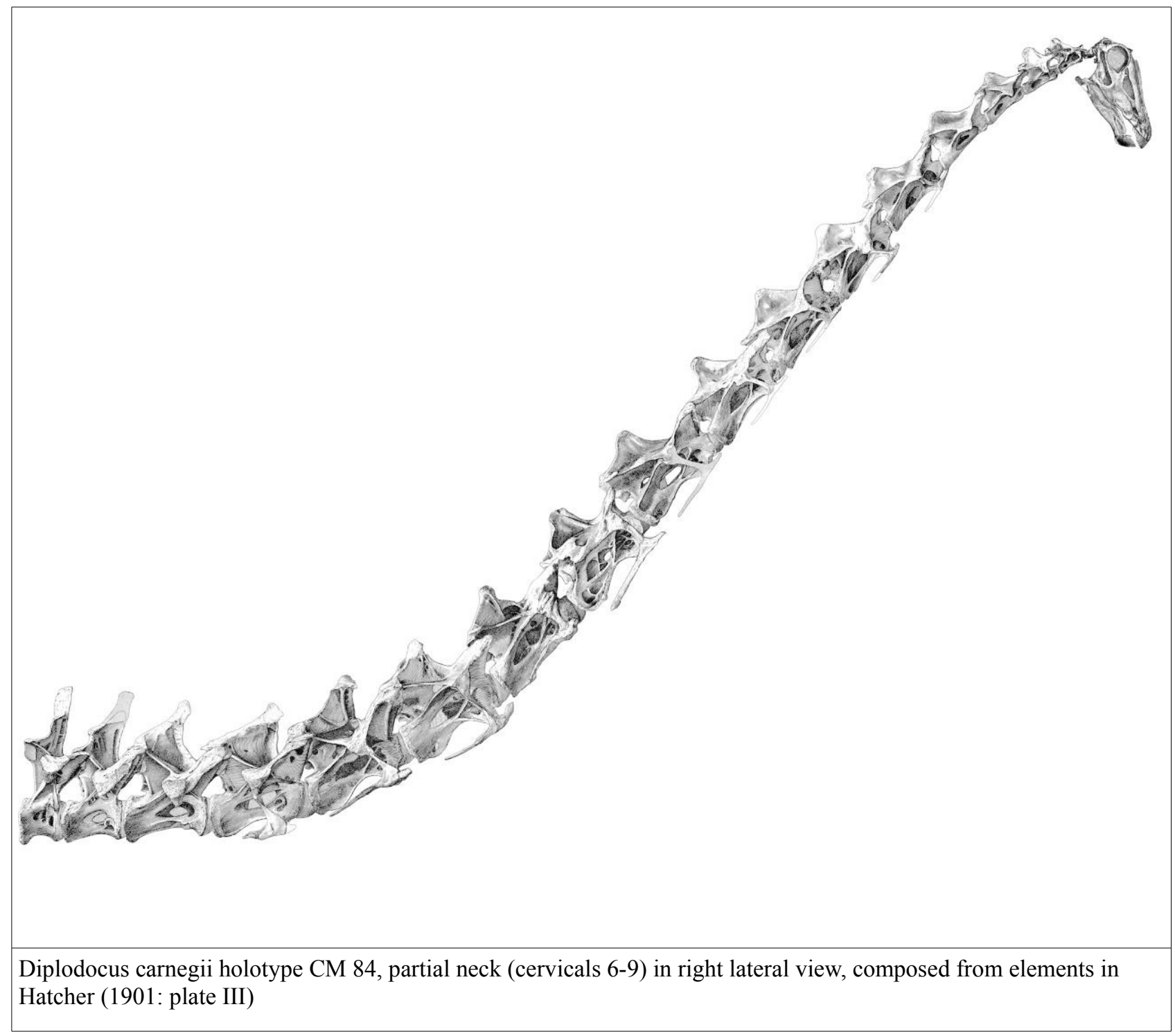

You'll see that, while the condyles are sat nicely in the cotyles, the zygapophyses are not at all well articulated: in particular, the C7-C8 and C8-C9 junctions have the prezygs shoved much too far forward, so that a double downward kink would be necessary to accomodate these articulations without pulling the condyles out of the cotyles.

Finally, while Matt and I were in Berlin last November, as part of the excursion associated with the awesome all-sauropod-gigantism-all-the-time workshop, we got to play with the superbly preserved set of anterior brachiosaur cervicals HMN SI, and we tried to articulate the real bones. We had to stop for fear of breaking them, because they simply would not fit nicely together.

In conclusion, the distortion of all sauropod cervicals renders them poor subjects for quantitative analysis such as that of the DinoMorph project. While the approach of Stevens and Parrish is a real and valuable contribution to rigour in the analysis of posture, the output of DinoMorph is a 
hypothesis to be tested by other lines of evidence rather than a firmly established fact. (That last bit was quoted verbatim from our paper.)

I've gone on much longer than I intended to in what was supposed to be a quick-and-easy post, so I'll leave it here. In order to keep the recent paper short and snappy, we didn't go into this in much detail, summarising down to a mere 88 words (Taylor et al 2009: 216-217), so maybe this will bear repeating (in more rigorous form) in a future publication.

\section{References}

- Gilmore, C.W. 1936. Osteology of Apatosaurus with special reference to specimens in the Carnegie Museum. Memoirs of the Carnegie Museum 11: 175-300.

- Hatcher, J.B. 1901. Diplodocus (Marsh): its osteology, taxonomy and probable habits, with a restoration of the skeleton. Memoirs of the Carnegie Museum 1: 1-63 and plates I-XIII.

- Martin, J. 1987. Mobility and feeding of Cetiosaurus (Saurischia, Sauropoda) why the long neck? In: P.J. Currie and E.H. Koster (eds.), Fourth Symposium on Mesozoic Terrestrial Ecosystems, Short Papers, 154-159. Box-tree Books, Drumheller, Alberta.

- Stevens, K.A. 2002. DinoMorph: Parametric modeling of skeletal structures. Senckenbergiana lethaea 82(1): 23-34.

- Stevens, K.A. and Parrish, J.M. 1999. Neck posture and feeding habits of two Jurassic sauropod dinosaurs. Science 284: 798-800. [Free subscription required]

- Taylor, M.P., Wedel, M.J. and Naish, D. 2009. Head and neck posture in sauropod dinosaurs inferred from extant animals. Acta Palaeontologica Polonica 54(2): 213-220.

- Wedel, M.J., Cifelli, R.L. and Sanders, R.K. 2000. Sauroposeidon proteles, a new sauropod from the Early Cretaceous of Oklahoma. Journal of Vertebrate Paleontology 20, 109-114.

Note: the original posted version of this article is on the Sauropod Vertebra Picture of the Week blog at http://svpow.wordpress.com/2009/05/30/range-of-motion-in-intervertebral-joints-why-we-donttrust-dinomorph/ 